\title{
Fabrication and application of dimyristoyl phosphatidylcholine biomaterial-based nanocochleates dry powder inhaler for controlled release resveratrol delivery
}

\author{
A. J. Mali ${ }^{*}$ (D, P. A. Joshi ${ }^{2}$ C. Bothiraja ${ }^{1}$ and A. P. Pawar ${ }^{1}$
}

\begin{abstract}
Background: Resveratrol, a bioactive phytoconstituent, is used to treat chronic respiratory diseases. However, its clinical application was hampered due to its poor bioavailability. In the present study, controlled release of resveratrol loaded nanocochleate-based dry powder inhaler was investigated to improve its biopharmaceutical properties for pulmonary drug delivery. The in vivo toxicity study was performed in the healthy male albino Wistar rats by intracheal administration.

Results: Resveratrol loaded nanocochleate-based dry powder inhaler was prepared by lyophilizing the resveratrol loaded dimyristoylphosphatidylcholine sodium and calcium ion-based nanocochleates using mannitol as cryoprotectant. Resveratrol loaded nanocochleates showed a particle size and encapsulation efficiency of $329.18 \pm$ $9.43 \mathrm{~nm}$ and $76.35 \pm 3.65 \%$, respectively. Resveratrol loaded nanocochleate-based dry powder exhibited a particle size of $102.21 \pm 9.83 \mu \mathrm{m}$ and satisfactory flowability with initial burst release followed by extended release up to 96 $\mathrm{h}$. The in vitro drug deposition pattern using multistage cascade impactor showed 1.28-fold improvement in fine particle dose, and the in vivo toxicity potential by histopathological study in albino rats revealed safety of formulation.
\end{abstract}

Conclusions: Resveratrol loaded nanocochleate-based dry powder inhaler could serve as an efficient delivery system for the treatment of chronic respiratory diseases.

Keywords: Resveratrol, Biomaterial, Nanocochleates, Dry powder inhaler, Controlled release, Drug delivery, Cascade impactor

\section{Background}

Chronic respiratory diseases (CRDs) are one of the leading causes of death worldwide, characterized by airflow restriction, bronchoconstriction, emphysema, chronic inflammation, and mucus hypersecretion. The tobacco smoke, inhalation of noxious particles, air pollution, genetics, and occupational chemicals are the leading factors

\footnotetext{
* Correspondence: ashwinjmali@rediffmail.com

'Department of Pharmaceutics, Poona College of Pharmacy, Bharati Vidyapeeth (Deemed to be University), Erandwane, Pune, Maharashtra 411038, India

Full list of author information is available at the end of the article
}

which prominently contribute to the development of CRDs. At present, some of the most widespread CRDs are asthma, chronic obstructive pulmonary disease (COPD), and occupational lung diseases [1, 2]. Recently, as per the World Health Organization (WHO), there are more than 100 million people of all age groups suffering from CRDs across the globe [2]. Considering the impact of CRDs on a universal level, it is clear that this disease presents a major public health challenge which further impact on the quality of individual life. The present line of treatment for CRDs is symptomatic which does not control or cure the progression of the disease by 
restoring usual lung function. Further, for CRDs, a very narrow treatment alternatives are available at present conditions such as inhaled corticosteroids (e.g., budesonide, fluticasone), anticholinergics (e.g., tiotropium, ipratropium), and $\beta$-2- agonists (e.g., salmeterol, indacaterol, formoterol). In some cases, CRDs become refractory to inhaled corticosteroids and the treatment fails. More recently, few scientific reports have confirmed the postulation of oxidative injury in CRDs and a direct injury to epithelial cells by reactive oxygen species (ROS) and reactive nitrogen species (RNS). These situations are worsened in smokers, a few studies in the literature have shown that cigarette smoke and oxidative stress increase histone acetylation which leads to increased expression of inflammatory genes. Hence, oxidative stress can be a key pharmaceutical target for the treatment of CRDs and antioxidant molecules have the potential to reinstate the response to inhaled corticosteroids [1, 3, 4].

Recently published literature has demonstrated that polyphenolic phytoconstituents can regulate the production of ROS and RNS to control oxidative stress chronic inflammatory diseases $[1,5]$. Resveratrol (RES) is a polyphenolic phytoalexin. Chemically, it is 3,5,4'-trihydroxytrans-stilbene and isolated from the roots of Veratrum grandiflorum O. Loes (Melanthiaceae). It shows antioxidant potential by inhibiting ROS by activating AMPK (5'adenosine monophosphate-activated protein kinase). It displayed better anti-radical activity as compared to other polyphenols such as kaempferol, catechin, naringenin, myricetin, and $\alpha$-tocopherol which justifies its useful effects in oxidative stress-mediated disease conditions. RES is also well known for treatment of various intrapulmonary and extrapulmonary diseases, such as cystic fibrosis, COPD, pulmonary hypertension, and lung cancer. In spite of potent antioxidant activity, light-sensitive nature, short biological half-life ( $<15 \mathrm{~min})$, extensive first-pass metabolism, poor water solubility $(0.0688 \mathrm{mg} / \mathrm{mL})$, and high lipophilicity (log $\mathrm{P}$ of 3.06 ) are the major challenges for successful delivery RES in clinically [6-9].

Drug delivery techniques currently available for CRDs treatment are nebulizers, soft mist inhalers (SMIs), pressurized metered-dose inhalers (pMDIs), and dry powder inhalers (DPIs). Administration of drugs through nebulization is a helpful treatment but only for stationary use. The pMDIs are more useful but need good coordination between dose actuation and inhalation. The DPIs were developed to overcome the weaknesses of nebulizers and pMDIs. DPIs present better physicochemical stability, do not need cold-chain storage or reconstitution, and no coordination problem [10-12]. Furthermore, the powder is dispersed into fine particles by the patient's inspiratory flow, and generally, the device is inexpensive, thus suitable for the treatment of CRDs. A variety of molecules such as phytoconstituents, anticancer agents, proteins, and peptides can be delivered to the pulmonary region using DPIs for treatment of various intrapulmonary and extrapulmonary diseases such as COPD, bronchiectasis, tuberculosis, lung cancer, and pulmonary hypertension.

Nanocochleates (NC) are lipid-based supramolecular assemblies $[13,14]$. They are mainly made up of three constituents: the negatively charged phospholipid bilayers (liposomes), the multivalent metal ions, and the therapeutic agent to be delivered; on varying one or more of these elements, various permutation and combinations are feasible. NC has an elongated shape, cigar-like spiral rolls, or a carpet roll like morphology. They vary from liposomes in having a water-free inner core and rigid stable structure. These unique morphological properties make nanocochleates a great platform for the delivery of drugs [15]. NC structure is resistant to diffusion of oxygen and as a result less susceptible to oxidation of encapsulated agent. It also provides protection from degradation for an encapsulated agent in biological fluids. Since the whole $\mathrm{NC}$ structure is a series of lipid layers, components within the core of the NC structure remain intact, although its outer lipid layers may be exposed to harsh environmental surroundings. NC has demonstrated their capability by improving physicochemical, biopharmaceutical properties, and therapeutic efficacy of a variety of molecules such as phytoconstituents like fisetin, anticancer agents (paclitaxel, doxorubicin), vitamins (retinol), and also macromolecules. Therefore, it is of interest to improve the RES activity of antioxidant by developing NC drug delivery system [15-18].

The aim of the present study was to formulate RES loaded NC dry powder inhaler (RES-NC- DPI) to improve its physicochemical and biopharmaceutical properties. Using the trapping method, RES-loaded dimyristoylphosphatidylcholine sodium vesicles were converted into $\mathrm{NC}$ by addition of calcium ions. Besides, RES-NC was lyophilized using mannitol which acted as cryoprotectant as well as DPI carrier. Developed RESNC were examined for particle size, zeta potential, encapsulation efficiency, and surface morphology. Furthermore, the lyophilized RES- NC-DPI powder was characterized using powder X-ray diffraction (PXRD), differential scanning calorimetry (DSC), scanning electron microscopy (SEM), and powder flow properties. Additionally, RES-NC-DPI was studied for various physicochemical properties, in vitro drug release in phosphate buffer saline ( $\mathrm{pH}$ 7.4), in vitro drug deposition pattern using multistage cascade impactor, and in vivo toxicity potential in albino rats.

\section{Methods \\ Materials}

Trans-resveratrol (99\%) and dimyristoylphosphatidylcholine sodium (DMPC $\mathrm{Na}^{+}$) were obtained as a gift 
sample from Mega resveratrol, USA, and Lipoid GmbH, Germany, respectively. Cholesterol was purchased from Fine Chem Industries Ltd., India. Dialysis membrane bag (molecular weight cutoff $12,000 \mathrm{Da}$ ) was purchased from Sigma-Aldrich Pvt. Ltd., India. Inhalation grade lactose (Respitose ${ }^{\circ}$ ML001) was obtained from DFE Pharma, Germany. All other solvents and chemicals used were of analytical grade.

\section{Preparation of RES-loaded vesicle}

RES-loaded vesicles (RES-V) were prepared according to the previously published method with slight modification $[17,19]$. As shown in Table 1, specific amount of DMPC sodium, cholesterol was dissolved in $3 \mathrm{~mL}$ of chloroform whereas RES was dissolved in $3 \mathrm{~mL}$ of ethanol and mixed together with heating to the phase transition temperature of DMPC sodium. The resulting mixture was rapidly injected into $10 \mathrm{~mL}$ deionized water under continuous stirring at $500 \mathrm{rpm}$ under vacuum until complete evaporation of chloroform and ethanol to obtained vesicles. The final volume was adjusted with deionized water. Lastly, prepared RES vesicles were purified by filtering through a $0.45-\mu \mathrm{m}$ membrane filter. The filtered RES-loaded vesicles were used to prepare the NC (Fig. 1).

\section{Preparation of RES-loaded nanocochleates (RES-NC)}

RES-NC was prepared by a previously reported method [15, 17]. A $10 \mu \mathrm{l}$ of $0.1 \mathrm{M}$ calcium chloride $\left(\mathrm{CaCl}_{2}\right)$ solution was added drop-wise into the RES-V under vortex. RES-V phase instantaneously turned opaque because of $\mathrm{NC}$ formation. Precipitated NC was refrigerated at $2-8^{\circ} \mathrm{C}$.

\section{Characterization of RES-NC}

The mean particle size and zeta potential were estimated by laser diffraction technique (Malvern 2000 SM; Malvern Instruments, Malvern, UK). The encapsulation efficiency was confirmed by the method reported by Bothiraja et al. [17]. Surface morphology of the RES-NC was determined using transmission electron microscopy (TEM; Philips CM- 200, Netherlands).

\section{Preparation of RES-NC dry powder inhaler}

RES-NC loaded dry powder inhaler (RES-NC-DPI) was prepared by lyophilization method. RES-NC dispersion was frozen at $-80^{\circ} \mathrm{C}$ for $24 \mathrm{~h}$ using mannitol as cryoprotectant and lyophilized using Free Zone freeze dryer (2.5 Liter Benchtop, Labconco, US) for $24 \mathrm{~h}$. Lyophilized cake was pass through sieve \# $140(105 \mu \mathrm{m})$ to obtain free flow RES-NC-DPI. The conventional-RES dry powder inhaler (RES-DPI) were prepared by the previously reported method [20-22]. The RES was sandwiched with $\alpha$-lactose monohydrate (Respitose $e^{\circ}$ ML001) in equal volumes and transfer to a glass vial. Glass vial $(10 \mathrm{~mL})$ was used for the blending and vortexed (Vortex Genie-2, Scientific Industries Ltd., USA) for $10 \mathrm{~min}$. After blending, RES-DPI was filled in individual glass vials and stored in a desiccated environment so as to protect it from moisture.

\section{Characterization of RES-NC-DPI}

The particle size was determined by laser diffraction technique (Malvern 2000 SM; Malvern Instruments, Malvern, UK). Powder X-ray diffraction (PXRD) studies were performed using X-ray diffractometer 2 (PW 1729, Philips, Netherlands). Thermal properties were studied using differential scanning calorimetry (DSC) coupled with an intercooler and STAR $^{\mathrm{e}}$ software (821e; MettlerToledo, Switzerland). Surface morphology was studied using scanning electron microscopy (SEM; Cambridge Stereoscan S120, Cambridge, UK) at $\times 2000$ magnification. Besides, flow properties of prepared powders were characterized in terms of bulk density, tap density, angle of repose $(\Theta)$, Carr's index $(\mathrm{CI})$, and percent porosity by previously reported method [23-25].

Further, percent porosity $(\varepsilon)$ is used to determine compressibility of powder which is the degree of volume reduction due to an applied pressure is measurement of porosity changes during compaction and is calculated using following formula

$$
\mathrm{E}=1-\left(\frac{\mathrm{Pb}}{\mathrm{Pt}}\right) \times 100
$$

where $\mathrm{Pb}$ and $\mathrm{Pt}$ are bulk and taped density of DPI.

Table 1 Physicochemical characteristics of RES-loaded vesicles (RES-V) and RES-loaded nanocochleates (RES-NC) (mean \pm SD, $n=3$ )

\begin{tabular}{lllllll}
\hline Formulation & RES $(\mathbf{m g})$ & DMPC $(\mathbf{m g})$ & Cholesterol $(\mathbf{m g})$ & EE $(\%)$ & Particle size $(\boldsymbol{\mu m})$ & Zeta potential $(\boldsymbol{\mu m})$ \\
\hline RES-V1 & 1 & 60 & 20 & $61.42 \pm 2.82$ & $193.65 \pm 13.62$ & $-31.44 \pm 1.80$ \\
RES-V2 & 3 & 60 & 20 & $72.26 \pm 3.34$ & $206.12 \pm 14.12$ & $-38.12 \pm 2.71$ \\
RES-V3 & 6 & 60 & 20 & $59.61 \pm 3.12$ & $277.77 \pm 11.32$ & $-35.89 \pm 3.34$ \\
RES-NC & 3 & 60 & 20 & $76.35 \pm 3.65$ & $329.18 \pm 9.43$ & $-35.40 \pm 3.52$ \\
\hline
\end{tabular}

All the determinations performed in triplicate and values are expressed as mean Data are Mean \pm SD $(n=3)$ 


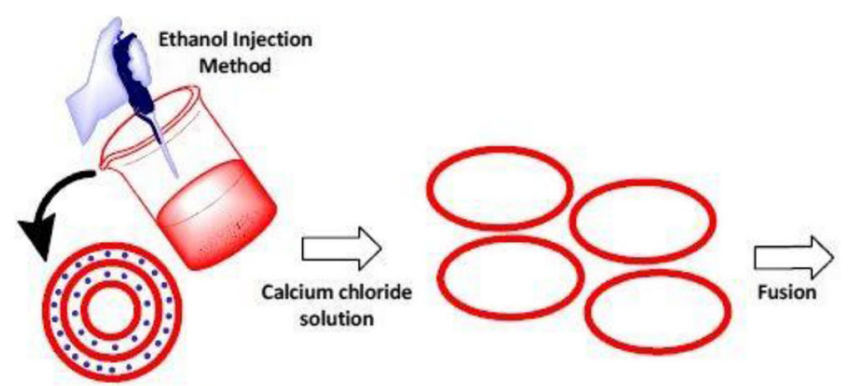

(A) Formation of multilaminar

(B) Formation of unilaminar lipid vesicles lipid vesicles
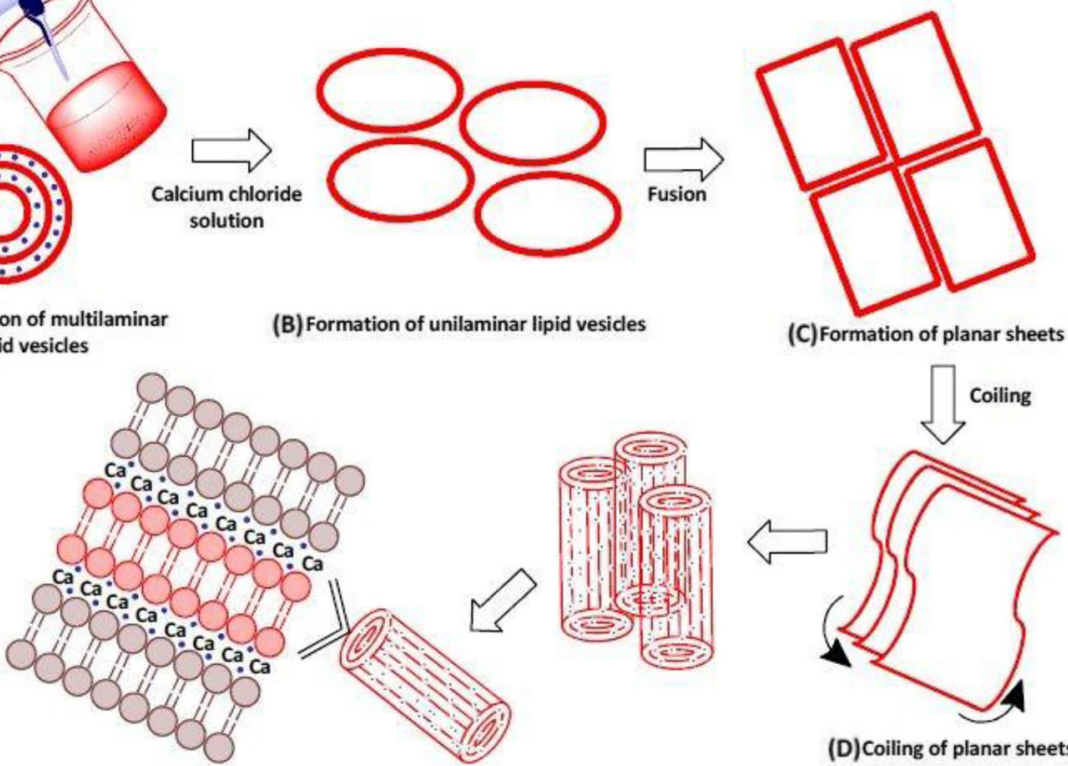

(E) Formation of rod shaped nanocochleates

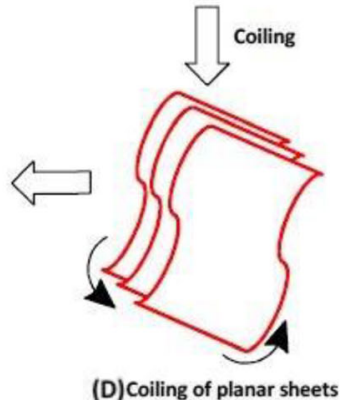

Fig. 1 Scheme for the synthesis of resveratrol loaded nanocochleates (RES-NC)

\section{In vitro release study}

The in vitro release of RES from RES-DPI and RES-NCDPI was investigated at physiological $\mathrm{pH}$ of 7.4 and temperature of $37 \pm 0.5^{\circ} \mathrm{C}$. The dialysis bag having molecular weight cutoff $12,000 \mathrm{Da}$ was used to carry out diffusion study. Briefly, formulation equivalent to 400 mcg of RES was loaded into a dialysis membrane bag, hermetically closed, and immersed into $100 \mathrm{~mL}$ of phosphate buffer saline with a $\mathrm{pH}$ of 7.4. The whole system was kept at $37 \pm 0.5^{\circ} \mathrm{C}$ with constant stirring at 100 $\mathrm{rpm} / \mathrm{min}$. Aliquots of $1 \mathrm{~mL}$ were withdrawn at predetermined time intervals and replaced with the same volume of fresh medium to retain constant volume. The withdrawn aliquots were filtered through Whatman filter paper $(0.45 \mu \mathrm{m})$, and the amount of RES in the aliquots was quantified using a UV-VIS absorption spectrophotometer (Shimadzu UV-1601, Japan) at 306 $\mathrm{nm}$ after suitable dilution. Cumulative RES release amount at pre-determined time intervals was mapped as the percentage released versus the time. The RES release mechanism from RES-NC-DPI and RES-DPI was analyzed by different kinetics models such as zero-order release $M_{t} / M_{\infty}=k_{0} t$, first-order release $\mathrm{M}_{\mathrm{t}} / \mathrm{M}_{\infty}=1-\exp \left(-\mathrm{k}_{1} \mathrm{t}\right)$, and Higuchi $\mathrm{M}_{\mathrm{t}} / \mathrm{M}_{\infty}=$ $\mathrm{k}_{\mathrm{H}} \mathrm{t}^{1 / 2}$ equation. The data analysis was performed using PCP Disso software (V3; Poona College of Pharmacy, Pune, India). The results of triplicate measurements and their means were reported.

\section{In vitro deposition study}

In vitro drug deposition pattern of RES from RESDPI and RES-NC-DPI was determined using an eightstage, non-viable Andersen cascade impactor (ACI, Westech Instruments, UK). The ACI was equipped with USP induction port (IP) and pre-separator (PS), which was coupled to a vacuum pump and a critical flow controller (Westech Instruments, UK). Prior to analysis, the pre-separator was placed with $15 \mathrm{~mL}$ of methanol for sample recovery and the ACI plates were coated with glycerol containing silicone oil (1\%, $\mathrm{w} / \mathrm{v}$ ) to ensure efficient sample collection. Accurately weighed $25 \mathrm{mg}$ of each formulation (equivalent to 400 mcg of RES) was filled into size 3 hard gelatin capsules under controlled environmental conditions. For each trial, the formulation was actuated into the ACI at a constant flow rate of $60 \mathrm{~L} / \mathrm{min}$ via a Rotahaler ${ }^{\circ}$ inhaler device $(n=3)$. Following aerosolization testing, the ACI was dismounted and each plate was washed with a known volume of methanol. The amount of powder deposited at different stages was measured using a UV-VIS absorption spectrophotometer (Shimadzu UV-1601, Japan) at $306 \mathrm{~nm}$ after suitable dilution. The aerosolization efficiency was characterized in terms of emitted dose (ED), fine particle size (FPD), mass median aerodynamic diameter (MMAD), and geometric standard deviation (GSD). Additionally, the effective inhalation index (EI) and 
dispersibility was measured using the following equation

$$
\mathrm{EI}=E M+F P F
$$

where EM is the percent ratio of the ED to RD and FPF is fine particle fraction

$$
\mathrm{EI}=\sqrt{ } \mathrm{FPD}^{\mathrm{ED}} \times 100
$$

\section{In vivo toxicity assessment in rats}

Healthy male Wistar albino rats (200-250 g) were purchased from the National Toxicology Centre, Pune. Animals were housed under a controlled environmental condition $\left(25 \pm 2{ }^{\circ} \mathrm{C}, 12\right.$-h dark-light cycle) with free access to pellet diet (Nutri Vet Life Science, India) and water before experiments. During this adaptation phase, the health status of the rats was supervised daily. Animals were fasted overnight but offered free access to water before the initiation of the experiment. In vivo toxicity studies were carried out using a previously published method. Briefly, healthy male Wistar albino rats were randomly divided into three groups $(n=6)$.

The animals were observed continuously for the first $2 \mathrm{~h}$ and $24 \mathrm{~h}$ to identify the behavioral changes. However, there are no noticeable behavioral changes and mortality occurred. The acute toxicity assessment was performed to check the acceptability of the $\mathrm{NC}$ for the route of inhalation. Group I (control group) did not receive any treatment. Group II received a single intratracheal dose of RES-NC- DPI (equivalent to $400 \mathrm{mcg}$ of RES) and group III received a single intratracheal dose of RES-DPI (equivalent to $400 \mathrm{mcg}$ of RES). The suturing was carried out after dosing at the incision area. To prevent any infection, the povidone gel was applied. All groups are treated for 10 successive days. Twenty-four hours after the last treatment the animals were sacrificed by deep ether anesthesia, and the lung and trachea sections $(5 \mu \mathrm{m}$ thick) were collected and fixed in $10 \%$ formalin solution for histological examination. The obtained sections were stained with hematoxylin-eosin dye and examined under a light microscope (Olympus $\mathrm{CH02}$ ). Further, the performed study did not contain any hamane end-points.

\section{Statistical methods}

All the experiments were performed in triplicates. Data were recorded as mean \pm standard deviation. The statistical analysis was carried out using online Quick Calcs Graph Pad software. Data analyzed in following manner; ${ }^{*} p \leq 0.1$ (not statistical significant), ${ }^{* *} p \leq 0.01$ (statistical significant), ${ }^{* * * *} p \leq 0.001$ (very statistical significant), and $* * * * * 0.0001$ (extremely statistical significant).

\section{Results}

Particle size distribution

The particle size distribution of RES and RES-NC-DPI samples are shown in Table 2. The RES showed a mean particle size of $3.34 \pm 1.88 \mu \mathrm{m}$. Formulated RES-NC-DPI mean particle size of $102.21 \pm 9.83 \mu \mathrm{m}(p \leq 0.01)$, while Respitose $^{\oplus}$ ML001 and conventional RES-DPI showed a mean particle size of $126.88 \pm 11.43$ and $132.33 \pm$ $10.78 \mu \mathrm{m}$, respectively. The span value for all analyzed samples was $\leq 1.0$ representing uniform particle size distribution. Such uniform particle size distribution is useful in terms of efficacy of the DPI because high polydispersity of PSD might lead to high variations in drug deposition pattern.

\section{Powder X-ray diffraction studies}

PXRD analysis was performed to conclude the physical nature of RES within the final formulation. PXRD analysis results were shown in Fig. 2. The diffraction patterns of RES showed characteristic high-intensity diffraction peaks at $2 \theta$ values of $16.4^{\circ}, 19.3^{\circ}, 20.4^{\circ}, 22.5^{\circ}$, $23.6^{\circ}, 23.8^{\circ}, 25.3^{\circ}$, and $28.5^{\circ}$ signifying crystalline nature, while formulated RES-NC-DPI did not show any characteristic high-intensity diffraction peaks representing the amorphous nature of RES-NC-DPI [20, 26].

\section{Differential scanning calorimetry}

In addition to PXRD analysis, DSC study was performed to verify the physical nature of RES within the final formulation. The DSC thermograms of RES and RES-NCDPI are showed in Fig. 3. The DSC thermogram of RES displayed a single sharp endothermic peak at $267.7^{\circ} \mathrm{C}$ with the heat of fusion $46.8 \mathrm{~mJ} / \mathrm{mg}$ attributed to its melting point and confirming its crystalline nature, whereas no remarkable peak was observed in the DSC thermograms of RES-NC-DPI confirming its amorphous nature. DSC analysis shows that the crystalline RES was converted into the amorphous state.

\section{Scanning electron microscopy}

The scanning electron microscopy (SEM) of RES and RES-NC-DPI are shown in Fig. 4. The surface morphology of RES-NC-DPI appeared absolutely different from RES. The SEM exhibited morphology of RES-NC-DPI varied from irregular plate to smooth rod. The pure RES exhibited the existence of irregular rough plate-shaped particles with several asperities, whereas RES- NC-DPI showed uniform rod-shaped particles with a smooth surface. In addition, SEM analysis of the particle size is in good conformity with the laser sizing particle size analysis (Table 2). 
Table 2 Particle size and flowability properties of RES-NC-DPI and RES-DPI (mean \pm SD, $n=3$ )

\begin{tabular}{|c|c|c|c|c|c|c|c|}
\hline Formulation & Particle size $(\mu \mathrm{m})$ & Bulk density & Tapped density & Angle of repose $(\theta)$ & Carr's index & Hausner ratio & Percent porosity \\
\hline RES-NC-DPI & $102.21 \pm 9.83^{* *}$ & $0.083 \pm 0.01$ & $0.085 \pm 0.02$ & $31.72 \pm 2.12^{*}$ & $21.17 \pm 4.11^{*}$ & $0.97 \pm 0.01$ & $42 \pm 2.14$ \\
\hline RES-DPI & $132.33 \pm 10.78$ & $0.14 \pm 0.04$ & & & & & \\
\hline $0.24 \pm 0.12$ & $29.33 \pm 3.44$ & $16.24 \pm 3.16$ & $0.58 \pm 0.21$ & $3 \pm 1.11$ & & & \\
\hline RES alone & $3.34 \pm 1.88$ & - & - & - & - & - & \\
\hline Respitose $^{\oplus}$ (ML001) & $126.88 \pm 11.43$ & - & - & - & - & - & \\
\hline
\end{tabular}

All the determinations are performed in triplicate, and values are expressed as the mean data are mean \pm SD $(n=3)$;

${ }^{*} p \leq 0.1$ (not statistical significant), ${ }^{* *} p \leq 0.01$ (statistical significant) when compared to the control group RES-DPI

\section{Powder flow properties}

Powder flow properties were determined using static (CI) and a dynamic method (angle of repose). The considerable difference was observed in the powder flow properties RES-NC-DPI and RES-DPI. The values of CI , angle of repose , Hausner ratio, and porosity for RESNC-DPI and RES-DPI are given in Table 2. The flow properties of RES-NC-DPI and RES-DPI was within the acceptable limits $[25,27,28]$.

\section{In vitro release study}

In vitro release of the RES from RES-DPI and RES-NCDPI was studied by diffusion bag technique. The in vitro release study was carried out in phosphate buffer saline (pH 7.4) instead of simulated lung fluid (SLF) as its $\mathrm{pH}$ varies considerably with time. The in vitro release pattern of RES-DPI and RES-NC-DPI was showed in Fig. 5. RESDPI displayed around $30 \%$ RES release within the first 30 min, whereas RES release from RES-NC-DPI displayed a biphasic release pattern with initial burst release $(27 \%)$ within the first $2 \mathrm{~h}$ followed by controlled release up to 96 h (75.07 \%). Further, the release kinetic model results are depicted in Table 3. As per the release kinetics, RES-DPI and RES-NC-DPI best fitted $\left(R^{2}\right.$ values are 0.916 and 0.997) to the Higuchi kinetic model that indicated diffusion controlled release mechanism from the NCs.

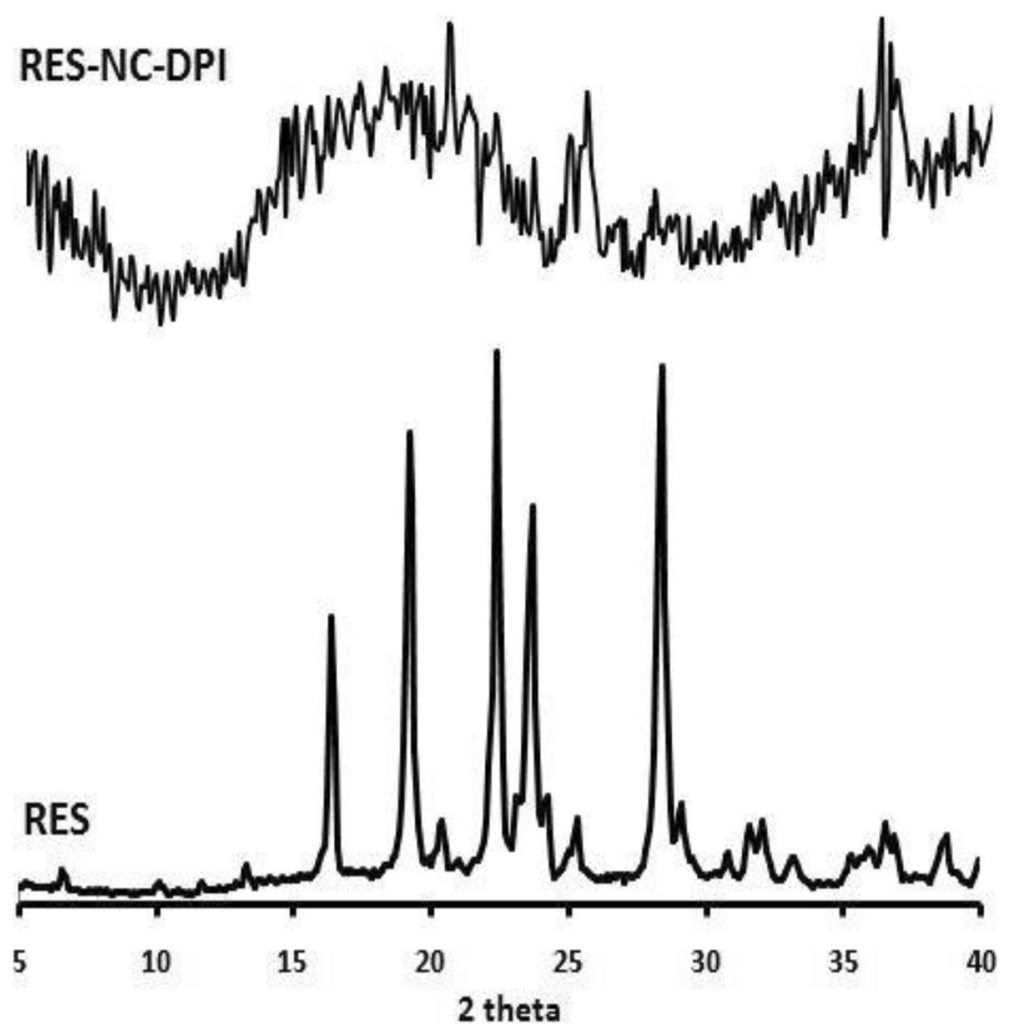

Fig. 2 Powder X-ray diffraction of RES and RES-NC-DPI 


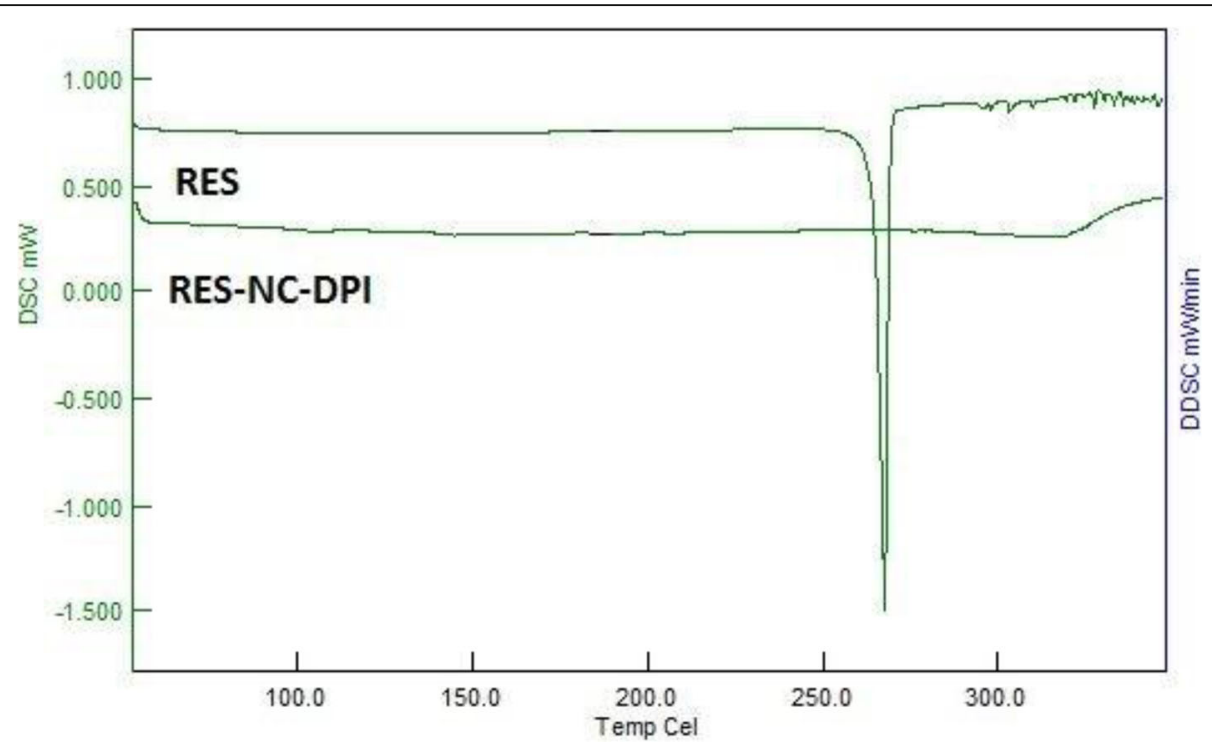

Fig. 3 DSC thermograms of RES and RES-NC-DPI

\section{In vitro deposition study}

The in vitro aerodynamic performance of RES-DPI and RES-NC-DPI was analyzed using ACI assembly equipped with USP IP and commercially available capsule-based inhaler device, i.e., Rotahaler (Cipla, India) at a constant flow rate of $60 \mathrm{~L} / \mathrm{min}$. Stage-wise deposition pattern in the ACI assembly for formulated RES-DPI and RES-NCDPI are showed in Fig. 6 and Table 4 display deposition profiles for RES actuated through the Rotahaler ${ }^{\circ}$ device. RES-DPI showed FPD and MB of $780.08 \pm 90.22 \mu \mathrm{g}$ and $89.94 \pm 7.00 \%$, respectively, whereas RES-NC-DPI displayed FPD and MB of $986.31 \pm 82.43 \mu \mathrm{g}$ and $91.60 \pm$ $8.33 \%$, respectively. RES-NC-DPI showed 1.26 -fold improvement in FPD as compared to RES-DPI $(p \leq 0.01)$. Besides, RES-DPI and RES-NC-DPI showed similar
MMAD of $2.30 \pm 0.99$ and $2.15 \pm 1.09 \mu \mathrm{m}$, respectively. Lower MMAD values exhibited suitable RES deposition pattern using the Rotahaler inhaler device. However, amounts of RES deposited within the non-sizing components (device, capsule, mouthpiece, IP and PS) were varied remarkably. Additionally, formulated RES-NC-DPI showed 1.21 and 1.22 fold higher percent recovery and emitted dose as compared to RES-DPI. These results indicated that RES-NC-DPI had effective aerodynamic performance most likely because of the particles smooth surface which control the cohesive-adhesive forces between the particles, therefore ease the aerosolization of DPI. Formulated RES-NC-DPI exhibited higher FPF of $54.87 \pm 9.32 \%$ as compared to previously reported spray dried resveratrol-PCL-microparticles (46.48 \%) , co-spray

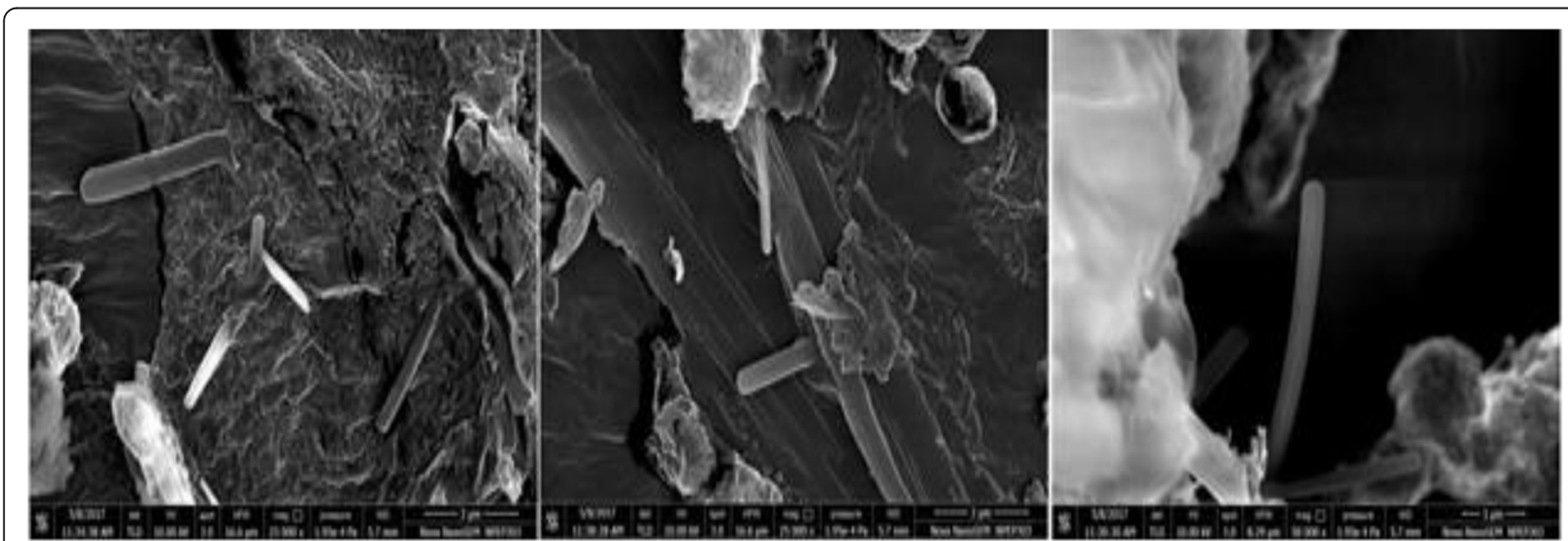

Fig. 4 Representative SEM micrographs of RES-NC-DPI 


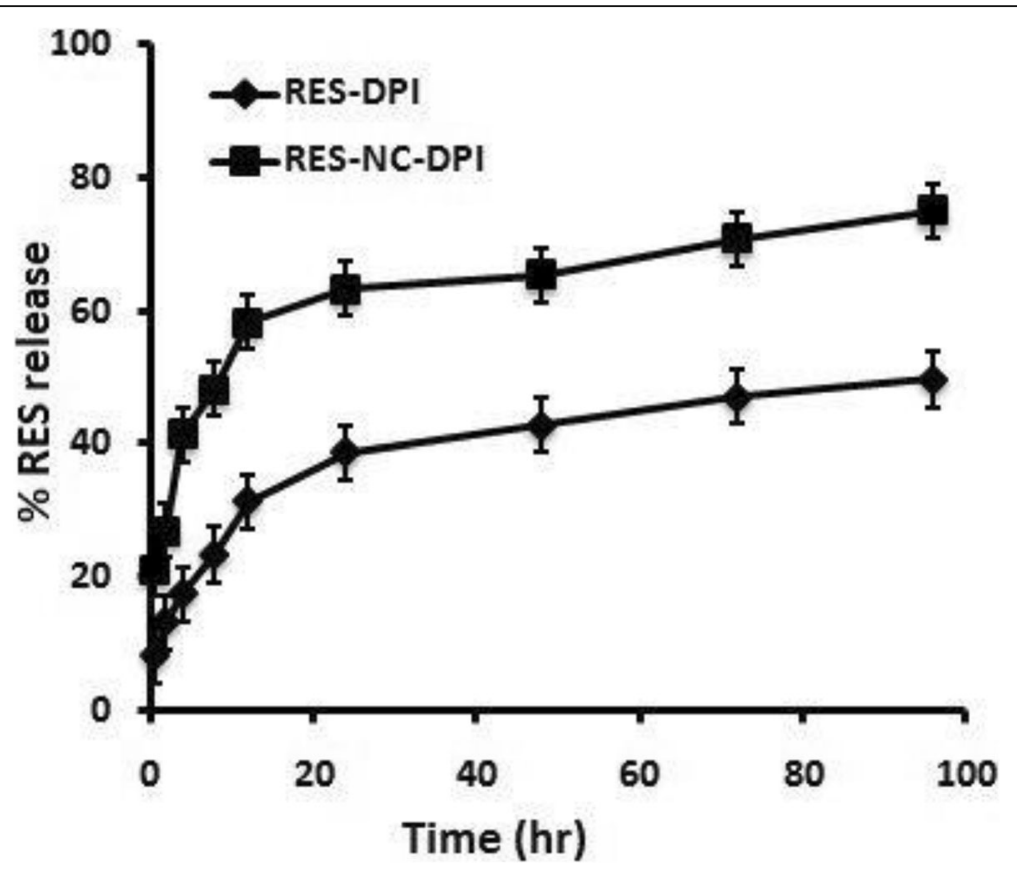

Fig. 5 In vitro release profile of RES alone and RES-NC-DPI in phosphate buffer saline (pH 7.4)

dried resveratrol and budesonide microparticles $(42.50$ $\%)[3,28]$, and spray dried resveratrol microparticles (39.90\%) [3].

\section{In vivo toxicity study}

RES is a well-known polyphenolic antioxidant. It plays an important role in chronic pulmonary diseases by controlling oxidative stress. But its clinical application can be limited by the risk of irreversible and perhaps life- threatening lung tissue injury. Thus, RES-DPI and RES-NC-DPI were tested for lung tissue toxicity in the Wistar rats by performing the macroscopic examination on lung and trachea. As shown in Fig. 7, RES-NC-DPI did not cause any significant tissue toxicity on the lung and trachea as compared to conventional RES-DPI. However, mild congestion was observed in the treated group which may be because of the installation procedure.

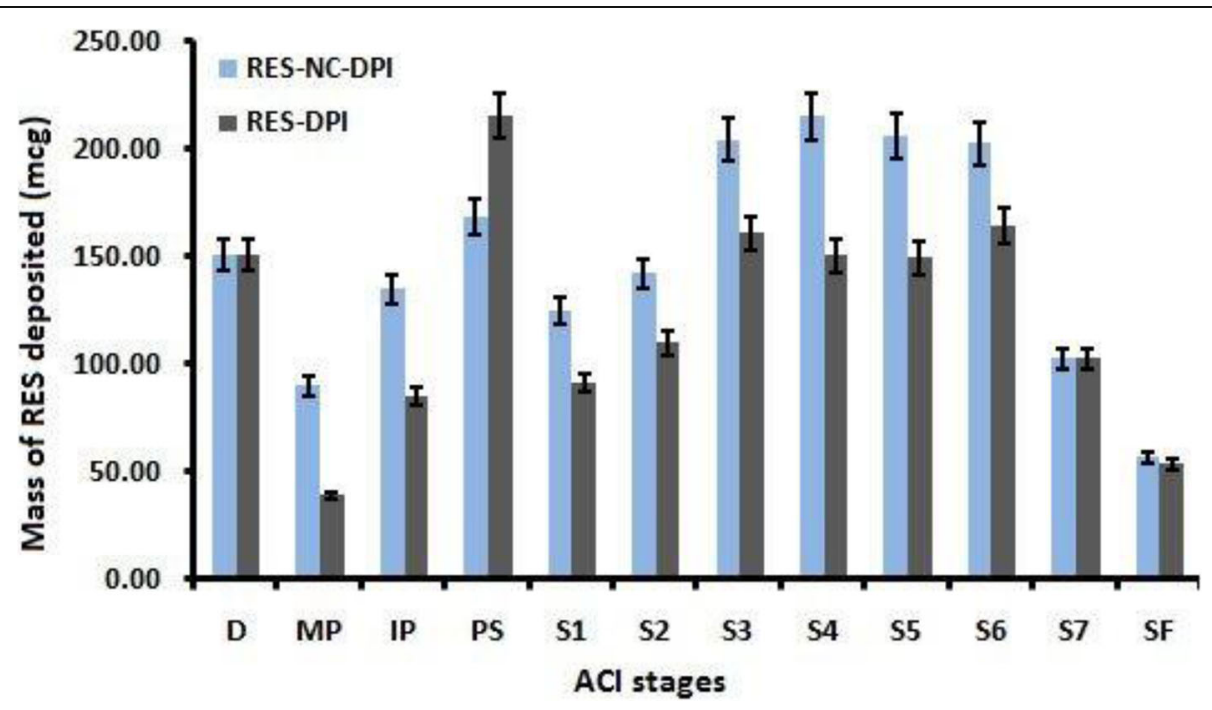

Fig. 6 Mass of RES deposited within the ACl assembly using the Rotahaler inhaler device at a flow rate of $60 \mathrm{~L} / \mathrm{min}$ 
Table 3 In vitro release kinetics in the cellophane membrane of RES-DPI and RES-NC-DPI

\begin{tabular}{llll}
\hline Formulation code & $\begin{array}{l}\text { Zero order } \\
r^{2}\end{array}$ & $\begin{array}{l}\text { First order } \\
r^{2}\end{array}$ & $\begin{array}{l}\text { Higuchi } \\
r^{2}\end{array}$ \\
\hline RES-DPI & 0.543 & 0.779 & 0.916 \\
RES-NC-DPI & 0.815 & 0.756 & 0.997 \\
\hline
\end{tabular}

Mean \pm SD $(n=3)$

\section{Discussion}

The RES-NC-DPI was developed to achieve the enhanced lung deposition and anticancer activity of RES by using the trapping method. Additionally, the lyophilized RES-NC-DPI was characterized for the physicochemical and biopharmaceutical properties along with the in vitro lung deposition by using the ACI.

Different acidic phospholipids are utilized to develop uniform sized and shaped vesicles [17, 29, 30]. In the present study, we preferred DMPC $\mathrm{Na}^{+}$as a lipid for vesicles formation because it is a vital constituent of the mammalian cell membrane and also shows superior compatibility for human use. With the aim of loading the maximum amount of RES into the lipid vesicles, vesicles were formulated to hold an increasing amount of RES (1, 3, and $6 \mathrm{mg}$ ) and analyzed for entrapment efficiency, particle size, and zeta potential. RES is a lipophilic bioactive, gets easily solubilized into the lipid phase of vesicles. At higher concentration of RES, though, the mean vesicle size increased but with lower entrapment efficiency because of exclusion of components from the lipid vesicles. The formulation RES-V2 represented satisfactory entrapment efficiency; therefore, the maximum amount that can be loaded into the vesicles was fixed at $3 \mathrm{mg}$.

RES-V2 lipid vesicles prepared by ethanol injection were transformed into $\mathrm{NC}$ using divalent cation. Various divalent cations $\left(\mathrm{Ca}^{2+}, \mathrm{Mg}^{2+}, \mathrm{Ba}^{2+}\right.$, and $\left.\mathrm{Zn}^{2+}\right)$ are reported for preparing $\mathrm{NC}$. It has been reported that $\mathrm{Ca}^{2+}$ forms a more greatly ordered, firmly packed and less hydrated arrangement than does $\mathrm{Mg}^{2+}$ with phospholipids. In addition, it is needed in much lower concentration than $\mathrm{Mg}^{2+}$. The addition of calcium ions to lipid vesicles stimulate fusion of lipid membrane and the development of planar sheets, which ultimately roll around a primary point of folding to form rod shape cylindrical NC. Furthermore, it is well reported that $\mathrm{Ca}^{2+}$ plays an essential role in natural membrane fusion phenomena whereas other cations noted above are ineffective in the majority of such systems. Therefore, it is most compatible with the body. Hence, $\mathrm{Ca}^{2+}$ is the most appropriate divalent cation for preparing $\mathrm{NC}$ and thus used in the present study [15].

The enhancement in the mean particle size was because of free RES entrapment and showed better entrapment efficiency as compared to RES-V2 vesicles. The reduction in the zeta potential than RES-V2 could be because of the interaction between $\mathrm{Ca}^{2+}$ and lipid resulting fractional neutralization heading to poor availability of anionic lipid on the surface. RES-NC were successfully lyophilized using 1-5\% mannitol as a cryoprotectant as well as the carrier (RES-NC-DPI). Mannitol is one of the most commonly used cryoprotectant and/or bulking agents as compared to other sugars. It helps to provide

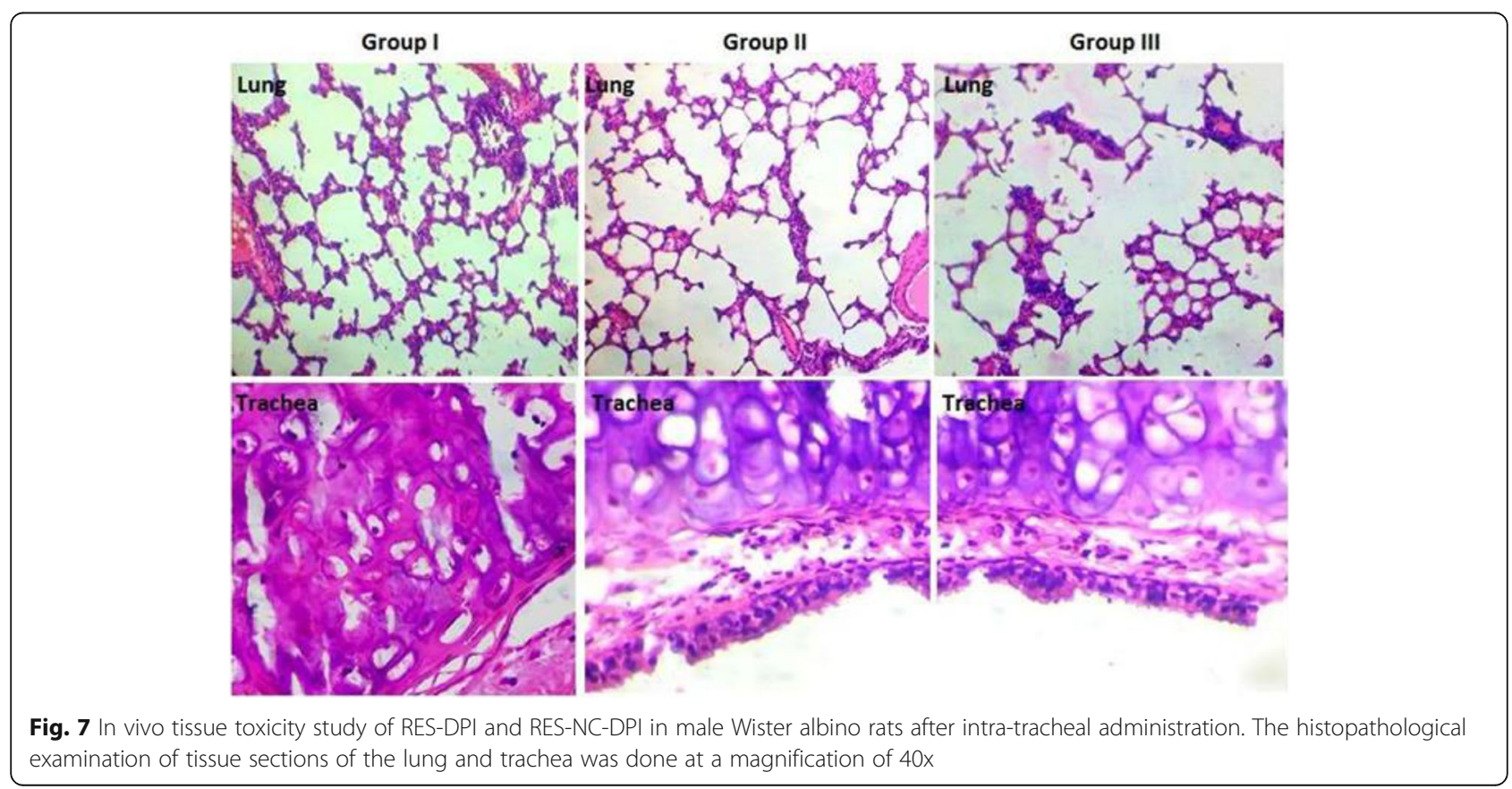


Table 4 Aerodynamic assessment of RES-NC-DPI and RES-DPI at a flow rate of $60 \mathrm{~L} / \mathrm{min}$ (mean $\pm \mathrm{SD}, n=3$ )

\begin{tabular}{lll}
\hline Aerodynamic parameters & RES-DPI & RES-NC-DPI \\
\hline RD $(\mu \mathrm{g})$ & $1470.67 \pm 110.22$ & $1797.50 \pm 109.65^{* *}$ \\
ED $(\mu \mathrm{g})$ & $1281.08 \pm 132.44$ & $1557.08 \pm 120.22$ \\
FPD $(\mu \mathrm{g})$ & $780.08 \pm 90.22$ & $986.31 \pm 82.43^{* *}$ \\
FPF $(\%)$ & $53.04 \pm 9.08$ & $54.87 \pm 9.32$ \\
MB $(\%)$ & $89.74 \pm 7.00$ & $91.60 \pm 8.33$ \\
MMAD $(\mu \mathrm{m})$ & $2.15 \pm 0.99$ & $2.30 \pm 1.09$ \\
GSD $(\mu \mathrm{m})$ & $3.60 \pm 1.22$ & $3.16 \pm 1.21$ \\
$D(\%)$ & $60.89 \pm 8.89$ & $63.34 \pm 9.77$ \\
El & $11.84 \pm 1.09$ & $11.90 \pm 1.03$ \\
\hline
\end{tabular}

$R D$ recovered dose, $E D$ emitted dose, $F P D$ fine particle dose, $F P F$ fine particle fraction, $M B$ mass balance, $D$ dispersibility, MMAD mass median aerodynamic diameter, GSD geometric standard deviation, and $E$ l effective inhalation index ${ }^{* *} p \leq 0.01$ (statistically significant) when compared to the control group RES-DPI

adequate structure to powder. The nature of lyophilized powder also depends on the ratio of drug and cryoprotectant $[29,31]$. PXRD of RES-NC-DPI confirms that the RES is fully integrated within the NCs $[20,26]$. The trapping method holds the lipid vesicles tightly and preserved the amorphous state of RES. The variation in the surface morphology and size of particles might change the aerodynamic deposition of the inhaled formulations.

As per the release data, the initial burst release may be because of the dissolution of free RES absorbed on the surface of the NCs, whereas the controlled release might be due to slow diffusion of RES from NC. The drug encapsulated into the spiral structure release slowly and needed a longer duration of time to release. The in vitro aerodynamic drug deposition behavior of RES-NC-DPI and RES-DPI was conducted using Anderson cascade impactor (ACI) assembly equipped with commercially

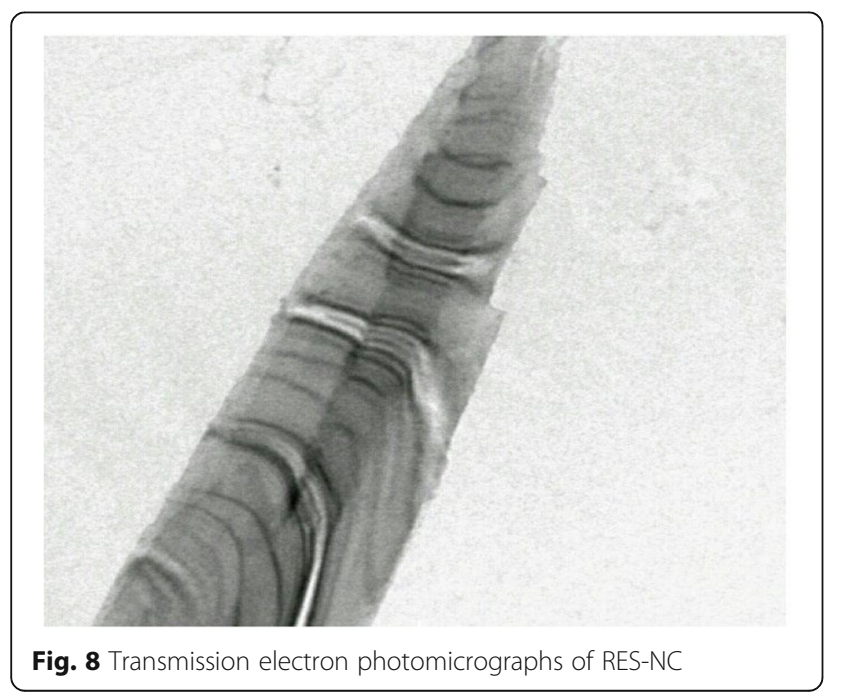

available inhaler device Rotahaler (low resistance device). In both cases, the amount of RES deposited on the nonsizing parts of the assembly, i.e., induction port (IP) and pre-separator (PS) varied immensely with the RES-DPI displaying a much better deposition in non-sizing part, whereas the RES-NC-DPI showed a better deep lung deposition characteristic with more than $50 \%$ deposition on the latter stages of the assembly. To sum it up, the deposition profile displayed by the RES-NC-DPI was far more superior than the RES-DPI with better deep lung deposition. The uniform rod-shaped particles with a smooth surface with tapering end may be the major contributing factor which results in the enhanced lung deposition (Fig. 8). This characteristic shape of the NCs was useful to increase not only the trajectory but also the in vitro aerodynamic efficacy to reach the deeper region of the lungs. Further, there was observed marked changes in the zeta potential of the vesicles and NCs. Initially, the higher end negative charges were observed on the vesicles in the preparation of NCs. But in the process of neutralization by using the divalent cation, there was a marked decrease in the zeta potential of the RES-NC-DPI which helpful in the spiraling of the NCs reflecting its cylindrical structure. Further, RES-NC-DPI has shown negative surface charge $-35.40 \pm 3.52 \mathrm{mV}$. The negative surface charge on the respiratory tract and RESNC-DPI was more prominently responsible for repulsive forces as per the charge theory which may increasing the time of flight and consequently lung deposition of the RES.

Further, the main reason behind the low toxicity for RES-NC-DPI could be accredited to the slow release of the RES from the NCs over a prolonged period of time which is released in 96-h duration as confirmed in in vitro release study. Briefly, results showed no signs of tissue toxicity in RES-NC-DPI-treated group representing its non-toxic nature. The results accomplished that developed novel formulation was safe for the pulmonary administration.

\section{Conclusion}

In the present study, RES-NC was successfully prepared by trapping method and transformed in the inhalable microparticles. In the case of RES-NC, a mixture of physiological lipid and cholesterol was used to form smooth surface rod-shaped NC and the lyophilized powders showed particle size, shape, and flow properties suitable for the deep lung deposition of RES. The RES-NC-DPI showed satisfactory aerodynamic performance with fine particle dose of $986.31 \mu \mathrm{g}$ vs $780.08 \mu \mathrm{g}$ for RES-DPI. Further, formulated RES-NC-DPI displayed controlled release up to $96 \mathrm{~h}$ in phosphate buffer saline (pH 7.4). A 
preliminary in vivo toxicity study showed no significant tissue toxicity in the lung and trachea. It still remains to be investigated whether the formulations effectively give in vivo deep lung deposition. Briefly, in depth in vivo study will be require so as to evaluate the deep lung deposition, pharmacokinetics performance, and antioxidant potential of these formulations.

\section{Abbreviations \\ CRDs: Chronic respiratory diseases; COPD: Chronic obstructive pulmonary disease; WHO: World Health Organization; ROS: Reactive oxygen species; RNS: Reactive nitrogen species; RES: Resveratrol; AMPK: 5'Adenosine monophosphate-activated protein kinase; SMIs: Soft mist inhalers; pMDIs: Pressurized metered-dose inhalers; DPIs: Dry powder inhalers: NC: Nanocochleates; RES-NC-DPI: RES loaded nanocochleate dry powder inhaler; PXRD: Powder X-ray diffraction; DSC: Differential scanning calorimetry; SEM: Scanning electron microscopy; DMPC $\mathrm{Na}^{+}$: Dimyristoylphosphatidylcholine sodium; ACl: Andersen cascade impactor; ED: Emitted dose; FPD: Fine particle size; MMAD: Mass median aerodynamic diameter; GSD: Geometric standard deviation; CPCSEA: Committee for the purpose of control and supervision of the experiment of animals; IAEC: Institutional Animal Ethics Committee; SLF: Simulated lung fluid}

\section{Acknowledgements}

The authors would like to express their heartiest thankfulness to Om sai Pharmaceuticals Ltd., Uttarakhand, India for Resveratrol gift sample. The authors would like to acknowledge the support from Bharati Vidyapeeth Deemed to be University, Poona College of Pharmacy, Pune, Maharashtra, India.

\section{Authors' contributions \\ We declare that this study was conducted by the authors named in this article. A J Mali: Data drafting and interpretation of the work and designing and presentation of the data. P A Joshi: The execution of the work and collection of the data. C Bothiraja: The data analysis and presentation. A P Pawar: The idea generation, analysis, and execution. The authors read and approved the final manuscript.}

\section{Funding}

There was no funding.

\section{Availability of data and materials}

The all data is available for the publication of research work upon request.

\section{Ethics approval and consent to participate}

A research proposal was prepared according to the guidelines of the Committee for the Purpose of Control and Supervision of the Experiment of Animals (CPCSEA). The experiment protocol was approved by the Institutional Animal Ethics Committee (IAEC) of Poona College of Pharmacy, Pune (CPCSEA/15/2015).

\section{Consent for publication}

Not applicable.

\section{Competing interests}

The authors declare that they have no competing interests regarding the present work.

\section{Author details}

'Department of Pharmaceutics, Poona College of Pharmacy, Bharat Vidyapeeth (Deemed to be University), Erandwane, Pune, Maharashtra 411038 , India. ${ }^{2}$ Department of Quality Assurance, Poona College of Pharmacy, Bharati Vidyapeeth (Deemed to be University), Pune, Maharashtra 38, India.
Received: 15 October 2020 Accepted: 19 January 2021

Published online: 15 February 2021

\section{References}

1. Trotta V, Lee WH, Loo CY, Haghi M, Young PM, Scalia S, Traini D (2015) In vitro biological activity of resveratrol using a novel inhalable resveratrol spray-dried formulation. Int J Pharm 491(1-2):190-197

2. Mehta P, Bothiraja C, Mahadik K, Kadam S, Pawar A (2018) Phytoconstituent based dry powder inhalers as biomedicine for the management of pulmonary diseases. Biomed Pharmacother 108:828-837

3. Trotta V, Lee WH, Loo CY, Young PM, Traini D, Scalia S (2016) Co-spray dried resveratrol and budesonide inhalation formulation for reducing inflammation and oxidative stress in rat alveolar macrophages. Eur J Pharm Sci 30(86):20-28

4. Mehta P, Bothiraja C, Kadam S, Pawar A (2018) Potential of dry powder inhalers for tuberculosis therapy: facts, fidelity and future. Artif Cells Nanomed Biotechnol 46(3):S791-S806

5. Kapetanovic IM, Muzzio M, Huang Z, Thompson TN, McCormick DL (2011) Pharmacokinetics, oral bioavailability and metabolic profile of resveratrol and its dimethyl ether analog, pterostilbene in rats. Cancer Chemother Pharmacol 68(3):593-601

6. Santos AC, Pereira I, Pereira Silva M, Ferreira L, Caldas M, Collado GM Magalhaes M, Figueiras A, Ribeiro AJ, Veiga F (2019) Nanotechnology-based formulations for resveratrol delivery: effects on resveratrol in vivo bioavailability and bioactivity. Colloids Surf B Biointerfaces 180(3):127-140

7. Jayan H, Maria ML, Sivakama SK, Sundari JA, Moses C (2019) Anandharamakrishnan, improvement of bioavailability for resveratrol through encapsulation in zein using electrospraying technique. J Funct Foods 57:417-424

8. Huang XT, Li X, Xie ML, Huang Z, Huang YX, Wu GX, Peng ZR, Sun YN, Ming QL, Liu YX, Chen JP, Xu SN (2019) Resveratrol: review on its discovery, antileukemia effects and pharmacokinetics. Chem Biol Interact 306:29-38

9. Lindner GDR, Khalil NM, Mainardes RM (2013) Resveratrol-loaded polymeric nanoparticles: validation of an HPLC-PDA method to determine the drug entrapment and evaluation of its antioxidant activity. Sci World J 24:506083

10. Patil-Gadhe A, Pokharkar V (2014) Single step spray drying method to develop proliposomes for inhalation: a systematic study based on quality by design approach. Pulm Pharmacol Ther 27:197-207

11. Eliana N, Bucala V, Ramirez-rigo MV (2014) New alginic acid - atenolol microparticles for inhalatory drug targeting. Mater Sci Eng 41:255-266

12. Zhu L, Li M, Liu X, Jin Y (2017) Drug-loaded PLGA electrospraying porous microspheres for the local therapy of primary lung cancer via pulmonary delivery. ACS Omega 2:2273-2279

13. Mehta PP, Ghoshal D, Pawar AP, Kadam SS, Dhapte-Pawar VS (2020) Recent advances in inhalable liposomes for treatment of pulmonary diseases: concept to clinical stance. J Drug Deliv Sci Technol 56:101509

14. Mehta $P(2018)$ Imagine the superiority of dry powder inhalers from carrier engineering. J Drug Deliv 2018;1-19

15. Pawar A, Bothiraja C, Shaikh K, Mali A (2015) An insight into cochleates, a potential drug delivery system. RSC Adv 5(99):81188-81202

16. Liu Z, Liu D, Wang L, Zhang J, Zhang N (2011) Docetaxel-loaded pluronic P123 polymeric micelles: in vitro and in vivo evaluation. Int J Mol Sci 2: 1684-1696

17. Bothiraja C, Yojana BD, Pawar AP, Shaikh KS, Thort UR (2014) Fisetin-loaded nanocochleates: formulation, characterisation, in vitro anticancer testing, bioavailability and biodistribution study. Expert Opin Drug Deliv 11(1):17-29

18. Lin $\mathrm{R}, \mathrm{Ng} \mathrm{LS}$, Wang CH (2005) In vitro study of anticancer drug doxorubicin in PLGA-based microparticles. Biomaterials 26:4476-4485

19. Mohtar N, Taylor KMG, Sheikh K, Somavarapu S (2017) Design and development of dry powder sulfobutylether- $\beta$-cyclodextrin complex for pulmonary delivery of fisetin. Eur J Pharm Biopharm 113:1-10

20. Dimer FA, Ortiz M, Pohlmann AR, Guterres SS (2015) Inhalable resveratrol microparticles produced by vibrational atomization spray drying for treating pulmonary arterial hypertension. J Drug Deliv Sci Technol 29:152-158

21. Sinha B, Mukherjee B, Pattnaik G (2013) Poly-lactide-co-glycolide nanoparticles containing voriconazole for pulmonary delivery: in vitro and in vivo study. Nanomed Nanotechnol Biol Med 9:94-104

22. Crcarevska MS, Dodov MG, Goracinova K (2008) Chitosan coated Ca-alginate microparticles loaded with budesonide for delivery to the inflamed colonic mucosa. Eur J Pharm Biopharm 68:565-578 
23. Mali AJ, Pawar AP, Bothiraja C (2014) Improved lung delivery of budesonide from biopolymer based dry powder inhaler through natural inhalation of rat. Mater Technol 29:350-357

24. Mali AJ, Bothiraja C, Purohit RN, Pawar AP (2017) In vitro and in vivo performance of novel spray dried andrographolide loaded scleroglucan based formulation for dry powder inhaler. Curr Drug Deliv 14(7):968-980

25. Mali AJ, Pawar AP, Purohit RN (2014) Development of budesonide loaded biopolymer based dry powder inhaler: optimization, in vitro deposition, and cytotoxicity study. J Pharm 2014:795371

26. Karthikeyan S, Prasad RR, Ganamani A, Balamurugan E (2013) Anticancer activity of resveratrol-loaded gelatin nanoparticles on $\mathrm{NCl}-\mathrm{H} 460$ non-small cell lung cancer cells. Biomed Prev Nutr 3:64-73

27. Sehgal P, Gupta R, Singh U, Chaturvedi A, Gulati A, Sharma M (2012) Fast dissolving tablets : a new venture in drug delivery. Am J PharmTech Res 2: 110-119

28. Swaminathan S, Pastero L, Serpe L, Trotta F, Vavia P, Aquilano D, Trotta M, Zara G, Cavalli R (2010) Cyclodextrin-based nanosponges encapsulating camptothecin: physicochemical characterization, stability and cytotoxicity. Eur J Pharm Biopharm 74:193-201

29. Viswanathan V, Pharande R, Bannalikar A, Gupta P, Gupta U, Mukne A (2019) Inhalable liposomes of Glycyrrhiza glabra extract for use in tuberculosis: formulation, in vitro characterization, in vivo lung deposition, and in vivo pharmacodynamic studies. Drug Dev Ind Pharm 45(1):11-20

30. Levet V, Rosière R, Merlos R, Fusaro L, Berger G, Amighi K, Wauthoz N (2016) Development of controlled-release cisplatin dry powders for inhalation against lung cancers. Int J Pharm 515:209-220

31. Zhang T, Chen Y, Ge Y, Hu Y, Li M, Jin Y (2018) Inhalation treatment of primary lung cancer using liposomal curcumin dry powder inhalers. Acta Pharm Sin B 8:440-448

\section{Publisher's Note}

Springer Nature remains neutral with regard to jurisdictional claims in published maps and institutional affiliations.

\section{Submit your manuscript to a SpringerOpen ${ }^{\circ}$ journal and benefit from:}

- Convenient online submission

- Rigorous peer review

- Open access: articles freely available online

- High visibility within the field

- Retaining the copyright to your article

Submit your next manuscript at $\boldsymbol{\nabla}$ springeropen.com 